\title{
Resolving the stratification discrepancy of turbulent natural convection in differentially heated air-filled cavities Part I: Reference solutions
}

A. Sergent ${ }^{\mathrm{a}, \mathrm{b}, *}$, S. Xin ${ }^{\mathrm{c}}$, P. Joubert ${ }^{\mathrm{d}}$, P. Le Quéré ${ }^{\mathrm{a}}$ J. Salat $^{\mathrm{e}}$, F. Penot ${ }^{\mathrm{e}}$

${ }^{a}$ CNRS, LIMSI, BP 133, 91403 Orsay, France

${ }^{\mathrm{b}}$ UPMC Univ Paris 06, F-75005, Paris, France

${ }^{\mathrm{c}}$ CETHIL, CNRS/INSA-Lyon/UCBL, 69621 Villeurbanne cedex, France

${ }^{\mathrm{d}}$ LEPTIAB, Université de La Rochelle,17042 La Rochelle cedex 1, France

${ }^{\mathrm{e}}$ LET-ENSMA, BP 40109, 86961 Futuroscope cedex, France

* Corresponding author.

Email address: sergent@limsi.fr(A. Sergent).

Preprint submitted to International Journal of Heat and Fluid Flow24 September 2010 


\begin{abstract}
:
The problem of the long established thermal stratification discrepancy between numerical and experimental results is investigated in a three-part paper. The first part establishes reference solutions by means of three-dimensional (3D) spectral direct numerical simulations of a buoyancy-driven flow $\left(R a_{H}=\right.$ $\left.1.5 \times 10^{9}\right)$. Two configurations of differentially heated air-filled cavity are considered: an idealized cavity (perfectly adiabatic cavity, PAC) and an Intermediate Realistic Cavity (IRC) making use of experimentally measured temperature distributions (Salat, 2004) on its top and bottom walls. The IRC flow structure as well as its associated rms fluctuations correspond to the experimentally observed flow dynamics. However both configurations keep resulting in a core thermal stratification value equal to 1.0 whereas experiments lead to a stratification of about 0.5. It is proved that this stratification paradox is neither related to three-dimensional effects nor to the experimental thermal distributions applied on the horizontal walls. Resolving this stratification discrepancy is the subject of the last two parts of this paper (Sergent et al., 2010; Xin et al., 2010).
\end{abstract}

Keywords: natural convection, differentially heated cavity, direct numerical simulation, benchmark solutions, turbulence, Chebyshev approximation

\title{
1 Introduction
}

We consider in this paper a Differentially Heated Cavity (DHC) for parameter values resulting in weakly turbulent flow. This configuration has been the subject of many studies in the past decades as it constitutes a canonical exercise for testing the performances of numerical codes and/or turbulence models. The early studies were performed in $2 \mathrm{D}$ but, with the continuously increasing capacities of computers, more and more are carried out in 3D (Dol and Hanjalić (2001); Tric et al. (2000); Soria et al. (2004); Salat et al. (2004); Trias et al. (2007); Baraghi and Davidson (2007), for example). The laminar regime in a DHC was numerically explored in the late 70's and during the 80's, (Mallinson and de Vahl Davis, 1977; de Vahl Davis and Jones, 1983; Le Quéré, 1991; Fusegi et al., 1993) and these studies agree to a very large extent for values of the Rayleigh number up to $10^{6}$, whatever the numerical method used.

The situation is not the same for transitional or weakly turbulent flows, that is for Rayleigh numbers of the order of $10^{9}-10^{10}$. After a few early attempts to provide turbulent solutions using $k-\epsilon$ modelling (see 
e.g. Markatos and Pericleous (1984) as an example), an attempt to define a numerical reference solution for turbulent natural convection in a 2D DHC was made at the EUROTHERM/ERCOFTAC conference held in Delft in 1992 (Henkes and Hoogendoorn, 1992). On this occasion, a numerical benchmark was proposed for an air-filled square DHC with adiabatic horizontal walls at a Rayleigh number of $5 \times 10^{10}$. Most of the contributions used a RANS $k-\epsilon$ approach with various turbulence models. Two 2D DNS (direct numerical simulation) solutions were provided, one by Paolucci (1990) for a Rayleigh number of $10^{10}$ and one by Xin and Le Quéré (1995) for a Rayleigh number of $10^{9.5}$. This comparison exercise pointed out that the RANS solutions displayed a large scattering. Later on, a "RANS reference solution" was defined as the average of the half contributions contained in the minimum interval. It is thus a compound solution of almost all the contributions as the contributions retained for one quantity (maximum velocity, for example) in the reference solution are not the same as those retained for another quantity (Nusselt number, for example). In particular, the "reference" dimensionless thermal stratification at the cavity center $\left(<S>=\frac{\partial\langle\theta\rangle}{\partial z}\left(\frac{1}{2}, \frac{1}{2}\right)\right)$ was set to $S=0.539$ (Henkes and Hoogendoorn, 1995). It happens that this value was close to the thermal stratification equal to $\langle S\rangle=0.38$ predicted by Paolucci (1990) (it turned out that the integration time of Paolucci's DNS was too short to reach the corresponding asymptotic flow regime). However several authors using 2D or 3D DNS or Large Eddy Simulations (LES) approaches (Xin and Le Quéré, 1995; Nobile, 2002; Sergent et al., 2003; Soria et al., 2004; Salat et al., 2004; Trias et al., 2010a) have reported dimensionless values of the stratification $\langle S\rangle$ of about 1 . In fact, $\langle S\rangle$ taking a valueclose to 1 is in continuity with the value observed in the separated boundary layer regime for steady solutions with $R a \sim 10^{8}$, as it is now well established (Janssen and Henkes, 1996; Le Quéré and Behnia, 1998).

More recently, new contributions using LES have provided numerical values of the thermal stratification which are in good agreement with experimental measurements of thermal stratification. For instance, Peng and Davidson (2001), dealing with the experimental cavity of Tian and Karayiannis (2000), have reported numerical results in good agreement with the experimental observations when performing a 3D LES using the measured temperature distributions on the top and bottom walls and either adiabatic or periodic Boundary Conditions (BCs) in the spanwise direction. This seems to indicate that combining 3D simulations with the measured temperature distributions on the top and bottom walls, is the key to the prediction of the thermal stratification in the cavity core. Dol 
and Hanjalić (2001) have also considered the measured temperature on the cavity walls as Dirichlet BCs when performing 2D and 3D RANS with either a second moment closure model or a modified $k-\varepsilon$ model. In their study, the rear and front walls are either passive or thermally controlled but an unusual condition has been applied to the horizontal walls: an isothermal cold bottom wall and an isothermal hot top wall have been used. They have reported complete 2D and 3D comparisons with the experimental data available for the different configurations considered. However, their results have proved to be very dependent on the turbulence model and their comparisons with experimental data are not completely satisfactory. The persistence of the variety of predicted flow structures may lead to the occurrence of multiple solutions exhibiting different symmetries, as it has been shown in the Rayleigh number range $10^{7}-10^{8}$ in a cubic cavity (de Gassowski et al., 2006).

At the same time a joint research program has been set up in order to understand the unexplained discrepancy observed on thermal stratification in the cavity core. This joint team work is based on three different but complementary approaches: experimental measurements, 3D DNS and LES. A new experiment (Salat et al., 2004) has been built up to revisit the early experimental studies of Mergui and Penot (1997). Numerically, 3D DNS using spectral methods have been carried out along with 3D LES using finite volumes, for parameters corresponding to the experimental configuration.

This part, the first of a three part paper, is aimed at presenting a complete set of reference numerical results (DNS) in a DHC with idealized BCs (perfectly adiabatic BCs or measured temperature on the horizontal walls). These results are meant to provide reliable data for analyzing the influence of horizontal walls BCs on the flow structure.

The second part of the paper (Sergent et al., 2010) defines several configurations with increasing levels of realism in the thermal BCs. The corresponding results of LES are compared. The increasing improvement in predicting the thermal stratification in the cavity core is shown which resolves the stratification paradox. The third part of the paper (Xin et al., 2010) will show that the main physical phenomenon responsible for the temperature distributions on the cavity walls is the wall radiation.

The remainder of the present paper is structured as follows: next section is devoted to the physical problem of interest, then the equations are given together with a brief description of the numerical methods. In section 3 , the results are presented laying the emphasis on the influence 


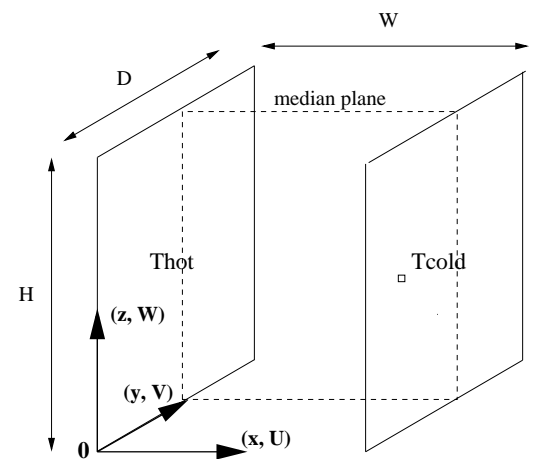

Fig. 1. The considered geometry.

of the thermal distribution on the horizontal walls. Comparison is based on qualitative and quantitative results for time-averaged flow, turbulent transition, power spectra and turbulent statistics are given. The conclusions are given in section 4.

\section{Physical problem and mathematical formulations}

\subsection{Geometrical configuration and thermal characteristics}

The geometrical configuration corresponds to an air-filled cavity of width $W$ in the $x$ direction, depth $D$, in the spanwise $y$ direction and height $H$ in the vertical $z$ direction as illustrated in Figure 1. Its two opposite vertical walls in the $x$ direction are maintained at uniform but different temperatures $T_{h}$ at $x=0$ and $T_{c}$ at $x=W$. Due to buoyancy, a fluid motion is induced in the cavity: it depends on the cavity geometry, the working fluid and the temperature difference, $\Delta T\left(=T_{h}-T_{c}\right)$. The front and rear walls are considered to be thermally insulated. Depending on the studied configuration, the two other walls (top and bottom walls) are either thermally insulated or maintained at a constant temperature distribution (see section 3.1).

In terms of dimensional analysis, the representative dimensionless parameters are the geometrical aspect ratios $\left(A_{x}=W / H=1, A_{y}=D / H=\right.$ $\left.0.32, A_{z}=1\right)$, the Prandtl number, $\operatorname{Pr}=\nu / \alpha(\operatorname{Pr}=0.71$ for air $)$ and the Rayleigh number, $R a=g \beta \Delta T H^{3} /(\nu \alpha)\left(R a=1.5 \times 10^{9}\right)$, where $\beta$ is volumetric thermal expansion coefficient, $g$ gravity acceleration, $\nu$ kinematic viscosity and $\alpha$ thermal diffusivity.

In the differentially heated cavity displayed in Figure 1, the buoyancy 
force results in a clockwise circulation which consists, at large enough Rayleigh number, in a thin upward vertical boundary layer along the hot wall and a thin downward boundary layer along the cold wall. The vertical boundary layers are connected by horizontal flows which take place along the top and bottom walls. The cavity core is mostly at rest and experiences a thermal stratification with a quasi-linear temperature distribution in the vertical direction. This thermal stratification can thus be characterized by the vertical temperature gradient at the cavity center: $S=\frac{\partial \theta}{\partial z}\left(A_{x} / 2, A_{y} / 2,0.5\right)$ where $\theta=\left(T-T_{0}\right) / \Delta T$ is reduced temperature with $T_{0}=\left(T_{h}+T_{c}\right) / 2$. In turbulent regime, it is defined in time-averaged sens with $\left\langle S>=\frac{\partial<\theta>}{\partial z}\left(A_{x} / 2, A_{y} / 2,0.5\right)\right.$ where the $<\cdot>$ denotes time averaging. Time-averaged heat transfer at the cavity walls is defined by the following Nusselt numbers:

- 1D Nusselt numbers averaged along the vertical and horizontal lines at the cavity mid-depth $\left(y=A_{y} / 2\right):<\overline{N u}_{1 D, h o t}>=\int_{0}^{1} \frac{\partial<\theta>}{\partial x}\left(0, A_{y} / 2, z\right) d z$ and $<\overline{N u}_{1 D, \text { cold }}>=\int_{0}^{1} \frac{\partial<\theta>}{\partial x}\left(A_{x}, A_{y} / 2, z\right) d z$

$<\overline{N u}_{1 D, \text { bottom }}>=\frac{1}{A_{x}} \int_{0}^{A_{x}} \frac{\partial<\theta>}{\partial z}\left(x, A_{y} / 2,0\right) d x$ and $<\overline{N u}_{1 D, \text { top }}>=$ $\frac{1}{A_{x}} \int_{0}^{A_{x}} \frac{\partial<\theta>}{\partial z}\left(x, A_{y} / 2,1\right) d x$

- 2D Nusselt numbers averaged over the vertical and horizontal walls:

$$
\begin{aligned}
& \left.<\overline{N u}_{2 D, h o t}>=\frac{1}{A_{y}} \int_{0}^{1} \int_{0}^{A_{y}} \frac{\partial<\theta>}{\partial x}(0, y, z) d y d z \text { and }<\overline{N u}_{2 D, \text { cold }}\right\rangle= \\
& \frac{1}{A_{y}} \int_{0}^{1} \int_{0}^{A_{y}} \frac{\partial<\theta>}{\partial x}(1, y, z) d y d z \\
& <\overline{N u}_{2 D, \text { bottom }}>=\frac{1}{A_{x} \times A_{y}} \int_{0}^{A_{x}} \int_{0}^{A_{y}} \frac{\partial<\theta>}{\partial z}(x, y, 0) d y d x \text { and }<\overline{N u}_{2 D, \text { top }}>= \\
& \frac{1}{A_{x} \times A_{y}} \int_{0}^{A_{x}} \int_{0}^{A_{y}} \frac{\partial<\theta>}{\partial z}(x, y, 1) d y d x
\end{aligned}
$$

Needless to note that adiabatic conditions along the top and bottom walls would imply zero Nusselt numbers there, although they are not equal to zero in the case of real experiments and in numerical simulations using measured temperature distribution on the top and bottom walls. 


\subsection{Governing equations}

Air in the cavity is considered to be newtonian and incompressible. Buoyancy-induced air flow is governed by the unsteady 3D Navier-Stokes under Boussinesq assumption in dimensionless form:

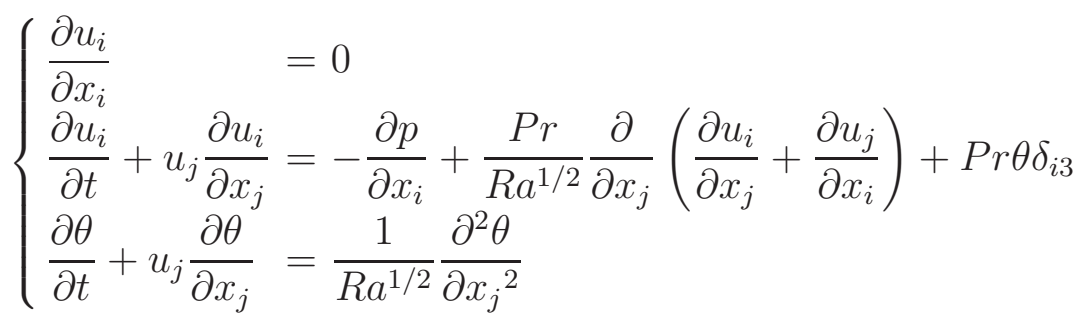

Dimensionless Equations 1 are obtained by using cavity height $(H)$ as reference length and the convective velocity $\left(\alpha R a^{1 / 2} / H\right)$ as reference velocity. $t$ denotes time, $x_{i}$ denotes the coordinates $\left(x_{i}=(x, y, z)\right), u_{i}$ is the velocity component in the $x_{i}$-direction $\left(u_{i}=(u, v, w)\right), p$ is the pressure and $\theta$, the reduced temperature, ranges from -0.5 on the cold wall to 0.5 on the hot wall.

In the present part of the three-part paper, the thermal BCs on the front/rear vertical walls $\left(y=0, A_{y}\right)$ are set to be adiabatic $(\partial \theta / \partial y=0)$. On the bottom/top horizontal walls $\left(z=0, A_{z}\right)$, thermal BCs can be either adiabatic or of Dirichlet type (measured distributions). No-slip $\mathrm{BCs}$ are set for velocity field on all 6 walls.

\subsection{Numerical methods}

The DNS code is based on a time marching procedure and uses spectral collocation methods as spatial discretization. Time integration of the governing equations is performed through a second-order semi-implicit scheme. It combines a second-order backward Euler scheme with an implicit treatment for the diffusion terms and an explicit second-order Adams-Bashforth extrapolation for the nonlinear terms. This time scheme results mainly in Helmholtz equations which should be solved at each time step. Incompressibility is imposed by a projection method which

retains second-order accuracy of the time integration (Guermond and Quartapelle, 1998; Achdou and Guermond, 2000). 
Helmholtz equations for velocity components and temperature, and pseudoPoisson equation for pressure correction are solved through total diagonalization of the discrete operators of second derivatives. Details of the method can be found in Xin and Le Quéré (1995; 2002).

With Chebyshev collocation methods, grid points are defined in each direction $(x, y, z)$ by the Gauss-Lobatto distribution:

$$
\xi_{i}=\left(1+\cos \left(\frac{i \pi}{N_{\xi}}\right)\right) \times \frac{A_{\xi}}{2} \quad ; \quad 0 \leq i \leq N_{\xi}
$$

where $A_{\xi}$ is the cavity aspect ratio in direction $\xi$.

In terms of wall units at $R a=1.5 \times 10^{9}$, the DNS grid results in $\Delta x^{+} \leq$ $0.2, \Delta y_{\max }^{+} \leq 0.2, \Delta z_{\max }^{+} \leq 0.4$ in the PAC case (see section 3.1 for definition).

\section{Reference numerical results}

\subsection{Investigated configurations}

We are interested in two configurations, one is academic with perfectly adiabatic passive walls and another is more realistic with measured temperature distributions on the horizontal walls. They are defined as follows:

- The academic configuration is a Perfectly Adiabatic Cavity (PAC) with two opposite vertical active walls and four other (top, bottom, front and rear) adiabatic walls. This configuration should be the canonical problem for both experimental and numerical investigations. However, since adiabatic walls are almost impossible when working with air, it becomes an idealized configuration which is only suited for numerical approaches.

- The more realistic configuration is named as Intermediate Realistic Cavity (IRC). It is also defined with two opposite vertical active walls and adiabatic front and rear walls. On the top and bottom walls, temperature distributions are considered to be known and independent of $y$. They correspond to the analytical fit of the measured temperature distributions along the centerline at $y=A_{y} / 2$ (Salat, 2004): 


\begin{tabular}{l|c|c|ccc} 
& Cavity type & Spatial resolution & Time step & Integ. time & Av. time \\
\hline$D N S_{P A C}$ & Perfectly Adiabatic & $180 \times 100 \times 200$ & 0.005 & 655 & 200 \\
$D N S_{I R C}$ & Intermediate Realistic & $180 \times 100 \times 200$ & 0.002 & 612 & 208 \\
\hline
\end{tabular}

Table 1

Numerical characteristics of the direct numerical simulations.

$$
\left\{\begin{array}{l}
\left.<\theta_{\text {bottom }}>(x, y)=(0.5-x)+0.994 \frac{x(x-1)(x-0.681)}{x(x-1)-0.0406(x+0.5(2)}\right) \\
<\theta_{\text {top }}>(x, y)=-<\theta_{\text {bottom }}>(1-x, y)
\end{array}\right.
$$

The IRC case relies on a close collaboration between experimental and numerical approaches and it is an important configuration toward resolving the stratification discrepancy of turbulent natural convection in differentially heated air-filled cavities.

Direct numerical simulations using spectral methods have been performed for the above configurations at $R a=1.5 \times 10^{9}$. They are aimed not only at understanding the corresponding flow regime but also at providing reference numerical solutions to both cases for the purpose of benchmark excercises. In a cubic PAC, Labrosse et al. (1997) and de Gassowski et al. (2003) have observed a first transition to unsteadiness at $R a_{c 1}=3.2 \times 10^{7}$ and de Gassowski et al. (2003)) have obtained several solution branches either steady or time-dependent up to $R a=10^{8}$. The Rayleigh number investigated is thus two decades higher than the first transition into unsteadiness in a cubic PAC. As can be seen below, flow is much more turbulent in the IRC than in the PAC, the DNS reference solutions of the IRC will be also used to demonstrate in the second part (Sergent et al., 2010) that the LES methodology is able to reproduce DNS results in a configuration which is more turbulent and closer to the experimental cell. The numerical parameters used in the simulations are reported in Table 1. As usual time integrations of the governing equations were started from a lower Rayleigh number and were carried out long enough for the flow regime and the turbulent statistics to become statistically established. In Table 1 the integration time is the time interval over which the governing equations have been integrated before starting the statistical sampling, expressed in units of dimensionless time. The averaging time is the period used for computing the turbulent statistics.

As said above, the present simulations of PAC and IRC have been performed with a Chebyshev spectral collocation approximation using 180 
points or polynomials in the horizontal direction, 200 points in the vertical direction and 100 points in the spanwise direction. Past benchmarks (de Vahl Davis and Jones, 1983; Janssen and Henkes, 1996) have shown that for the types of flow under consideration, it requires at least 5 more points in each direction with a finite difference approximation to achieve the same accuracy. Achieving the accuracy of the present results with typical finite difference codes would thus have required on the order of 1000 mesh points per direction, which has not been done so far. A further confirmation of the spatial accuracy of the presents results can be obtained by looking at the decay of the spectral coefficients of the temperature or velocity fields. A close inspection of these coefficients shows that they decrease with increasing order, and the respective ratios of $10^{5}$ for IRC and $10^{6}$ for PAC are consistently observed. Past studies (Le Quéré and Alziary de Roquefort, 1985) have shown that such values guarantee spatial convergence of the Chebyshev expansion.

The second point relates to the integration time, and to the fact that we have integrated long enough in time to reach the true asymptotic turbulent regime. It is well known that the asymptotic regime is reached after the transient effects induced by the sudden step change in Rayleigh number have died away. To make sure this is indeed the case, the equations were integrated for around 600 time units before starting computing statistical quantities. As it takes approximately 40 time units for the fastest fluid parcels to perform one lap in the cavity, a time length of 600 thus corresponds to approximately 15 times this global circulation time. Statistics were performed over the next 200 time units which corresponds to approximately 5 times that global circulation time. To our knowledge computations thus long have not been carried out before, except in the work of Trias et al. (2007).

An indirect quantification of the time needed to obtain accurate statistics can be obtained from checking that the time averaged solution obeys to the well known symmetries, in particular the reflexion symmetry about the middepth plane $y=A_{y} / 2$, although the instantaneous solution does not. The flow statistics reported hereunder (see Table 5) display this middepth symmetry to a very good approximation: the spatial deviation of the time-averaged transverse velocity field $(\langle V\rangle)$ to the theoritical value of zero has an averaged value on the mid-depth plane on the order of $10^{-6}$. This confirms that the averaging time used was sufficient for computing accurate flow statistics.

The combination of these two features, very large spatial resolutions and long time integrations, gives us confidence in the quality of the present 


\begin{tabular}{c|c|cc|cc} 
& $<S\rangle$ & $\left\langle\overline{N u}_{1 D, \text { hot-cold }}\right\rangle$ & $\left\langle\overline{N u}_{2 D, \text { hot-cold }}\right\rangle$ & $\left\langle\overline{N u}_{1 D, \text { bot-top }}\right\rangle$ & $<\overline{N u}_{2 D, \text { bot-top }}>$ \\
\hline$D N S_{P A C}$ & 1. & 61.1 & 60.1 & - & - \\
$D N S_{I R C}$ & 1. & 59.3 & 57.9 & 12.1 & 11.9
\end{tabular}

Table 2

Time-averaged thermal stratification $\langle S\rangle$ and Nusselt numbers for PAC and IRC.

results and allows us to claim their status of benchmark results.

\subsection{Heat transfer}

The PAC and IRC configurations differ only in the thermal BCs applied on the top and bottom walls. In IRC, these imposed temperature distributions result in a heat transfer between the fluid and the horizontal walls $\left(<\overline{N u}_{\text {bottom-top }}>\right)$. This additional heat transfer does not modify significantly the global Nusselt number on the vertical walls $\left(<\overline{N u}_{\text {hot-cold }}>\right)$ nor the central stratification $(<S\rangle$ ) (see Table 2$)$.

Despite the high value of the Rayleigh number, the overall Nusselt numbers along the vertical walls $\left.\left(<\overline{N u}_{2 D}\right\rangle\right)$ still fit the laminar $\overline{N u} \sim$ $0.30 R a_{H}^{1 / 4}$ relationship in both cases.

It is noted that the centerline Nusselt numbers $\left(\left\langle\overline{N u}_{1 D}\right\rangle\right)$ in both cases are slightly larger than the corresponding 2D Nusselt numbers. This is due to to the presence of the front and rear walls as already noted by Tric et al. (2000)) for steady flows in a cubic PAC. Although the global $\left\langle\overline{N u}_{1 D}>\right.$ is the same for both PAC and IRC cases, the mid-depth vertical profile of $\left\langle\overline{N u}_{1 D}>\right.$ is more uniform for IRC than PAC (Figure 2). This comes from the important effect of the thermal BCs applied on the horizontal top and bottom walls. In fact, thermal boundary conditions modified the global flow structure and the turbulence level in the vertical boundary layers as can be seen in next section.

\subsection{Time-averaged flow}

The sensivity of the flow structure to the thermal BCs applied on the bottom and top walls is shown on Figure 3. Due to the local heat transfer at the horizontal walls in the IRC, the horizontal flow along these walls is reinforced and experiences global reversal on the top and the bottom 


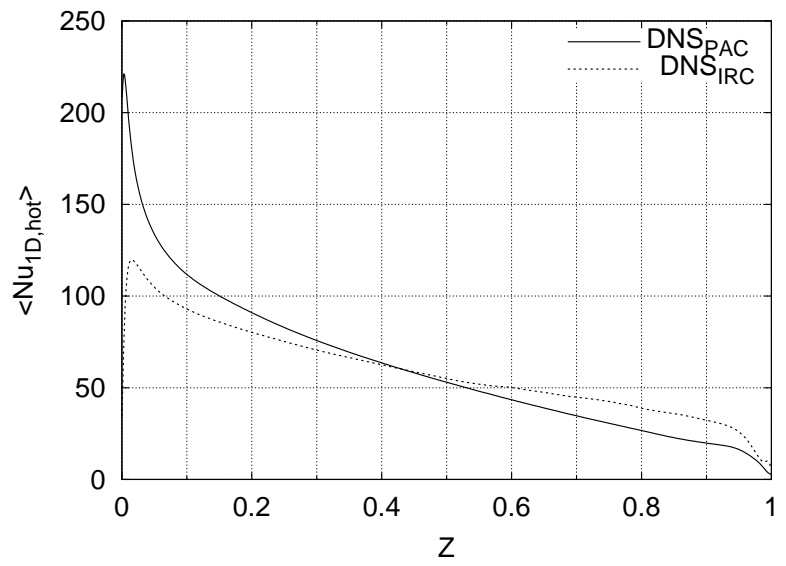

Fig. 2. Vertical profile of time-averaged Nusselt number along the hot wall in the mid-depth plane $\left(y=A_{y} / 2\right)$. Comparison between PAC and IRC.

parts of the cavity. This reversal has also been reported by several numerical studies Dol and Hanjalić (2001); Peng and Davidson (2001); Salat et al. (2004). They have shown that it was necessary to impose similar temperature distributions on the horizontal walls as that of experiments for reproducing the observed large flow reversal along the outer edge of the horizontal boundary layers (Tian and Karayiannis, 2000; Salat et al., 2004). This flow reversal does not exist in the PAC case, which displays the typical area of large eddies ejection instead (Xin and Le Quéré, 1995; Janssen and Henkes, 1996; Trias et al., 2007).

The IRC flow reversals create near the top and bottom walls two large areas which are more homogeneous in temperature than in the PAC. However the thermal stratification in the cavity core (approximatively for $z$ between $0.35-0.65)$ is not changed by the modification of the thermal BCs on the top/bottom walls, as can be seen on Figure 4.

The time-averaged characteristics of the velocity fields are gathered in Table 3 . In both cases, the flow remains mainly two-dimensional in the mid-depth plane, where the spanwise velocity component $(\langle V\rangle)$ values are weak. But for IRC, the magnitude of two horizontal velocity components $(\langle U\rangle,\langle V\rangle)$ is increased everywhere in the whole volume. This results from the aforementioned change of the flow structure, which affects not only the four boundary layers but also the cavity core.

Both cases differ substantially in the nearly motionless cavity core (Figure 3). In PAC, the upward and downward boundary layers entrain fluid through the whole cavity width respectively in the bottom and top half parts of the cavity core. In IRC, the strong flow reversals reduce by a 

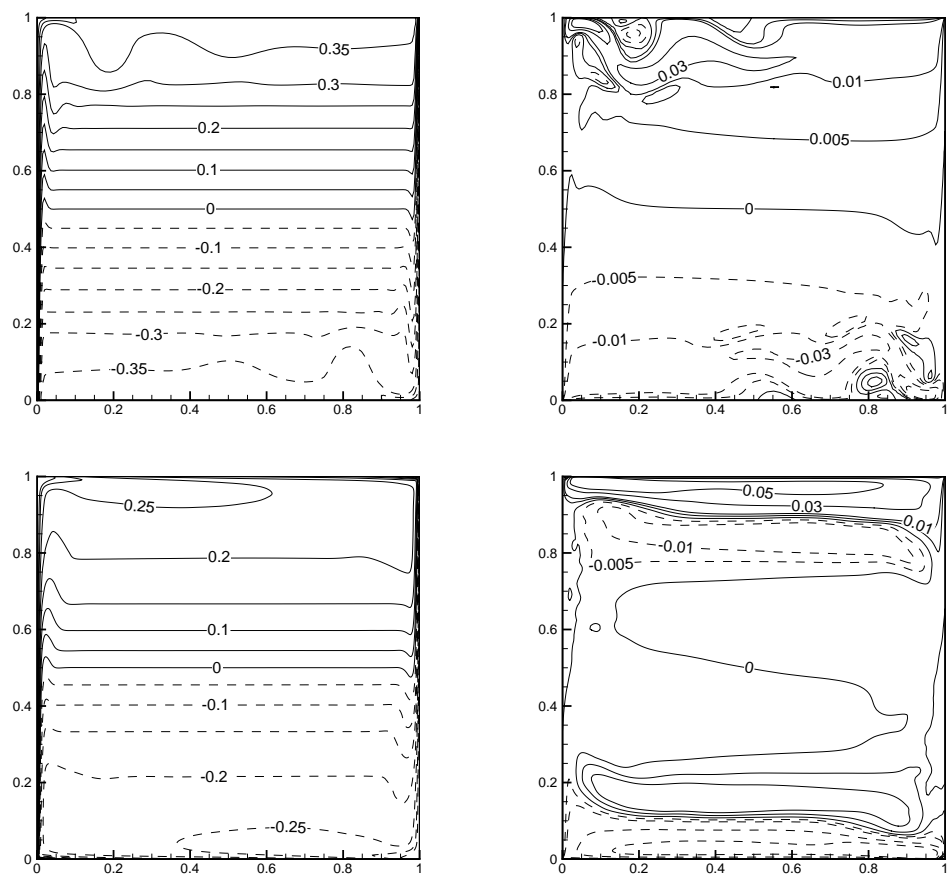

Fig. 3. Isocontours of temperature $\langle\theta\rangle$ (left) and horizontal velocity $\langle U\rangle$ (right) in the vertical mid-depth plane $\left(y=A_{y} / 2\right)$. Top: PAC ; bottom: IRC.
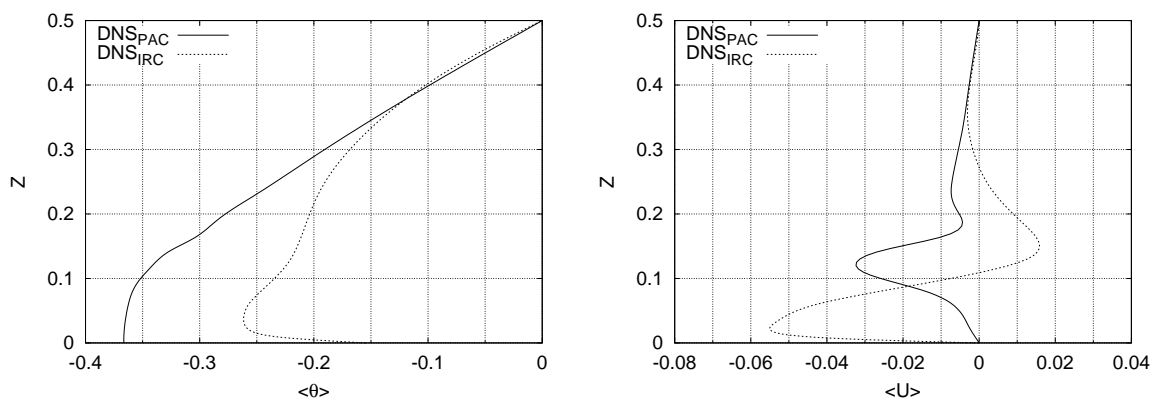

Fig. 4. Vertical profiles of time-averaged temperature $(\langle\theta\rangle)$ and horizontal velocity $(<U\rangle)$ along the vertical centerline $(x, y)=\left(A_{x} / 2, A_{y} / 2\right)$. Comparison between PAC and IRC.

factor two the height of the cavity core. The upward vertical boundary layer receives fluid mainly from the fast horizontal boundary layer up to the first quarter of the height $(z \sim 0.25)$, then from the cavity core approximatively up to $z \sim 0.6$.

At mid-height of the cavity $(z=0.5)$, the horizontal temperature profile (Figure 5) exhibits in both cases an undershoot temperature region at the outer edge of the boundary layer, which is a typical feature of thermal boundary layers in stratified medium (Yang et al., 1972). This 


\begin{tabular}{|c|c|c|c|c|c|}
\hline \multicolumn{3}{|c|}{$D N S_{P A C}$} & \multicolumn{3}{|c|}{$D N S_{I R C}$} \\
\hline$<U>_{\max }$ & $<V>_{\max }$ & $<W>_{\max }$ & $<U>_{\max }$ & $<V>_{\max }$ & $<W>_{\max }$ \\
\hline \multicolumn{6}{|c|}{ Maximum of velocity components on the entire volume } \\
\hline 0.0977 & 0.0294 & 0.2475 & 0.1130 & 0.0379 & 0.2571 \\
\hline$x=0.0468$ & 0.0272 & 0.0062 & $x=0.0760$ & 0.0245 & 0.0062 \\
\hline$y=0.0230$ & 0.3219 & 0.3184 & $y=0.2852$ & 0.3202 & 0.3202 \\
\hline$z=0.9938$ & 0.9961 & 0.4608 & $z=0.9925$ & 0.9970 & 0.4373 \\
\hline \multicolumn{6}{|c|}{ Maximum of velocity components on the mid-depth plane, $y=A_{y} / 2$} \\
\hline 0.0837 & 0.0014 & 0.2152 & 0.1069 & 0.0037 & 0.2175 \\
\hline$x=0.0545$ & 0.2887 & 0.0062 & $x=0.0760$ & 0.0148 & 0.0062 \\
\hline$z=0.9880$ & 0.9926 & 0.4764 & $z=0.9911$ & 0.1002 & 0.4373 \\
\hline \multicolumn{6}{|c|}{ Maximum of velocity components on the mid-height plane, $z=A_{z} / 2$} \\
\hline 0.0018 & 0.0027 & 0.2416 & 0.0064 & 0.0053 & 0.2543 \\
\hline$x=0.0432$ & 0.0092 & 0.0062 & $x=0.0301$ & 0.0218 & 0.0076 \\
\hline$y=0.0204$ & 0.2817 & 0.3184 & $y=0.0065$ & 0.2921 & 0.3184 \\
\hline
\end{tabular}

Table 3

Maximum of time-averaged velocity components for the PAC and IRC cases.

spatial oscillation is still present in the PAC case at $z=0.7$, whereas it disappears at this height in the IRC case owing to the flow reversal which interferes with the vertical boundary layer.

In the PAC, the fluid carried by the vertical boundary layers impinges the top or bottom wall and experiences several flow reversals downwards and upwards before being dragged along towards the opposite vertical wall. Figure 3 displays the two separated (recirculating) horizontal boundary layers with successive detachments and re-attachments in the top and bottom parts of the cavity. For the sake of brevity, these horizontal fluid layers are called horizontal separating fluid layers thereafter. The location of these separated regions can be evaluated from the horizontal distribution of the wall shear stress (Figure 6) approximatively at $x \sim 0.088-0.125$ for the first region and $x \sim 0.383-0.456$ for the second one. This figure also points out that the shear stress becomes slightly negative at $x \sim 0.21$, which is not visible in Figure 3 . As no separated region is present in the $I R C$ case, a quasi-constant wall shear stress $\left(\tau_{\text {wall }}\right)$ is generated in the center part of the horizontal walls $(0.3 \leq x \leq 0.8)$. 

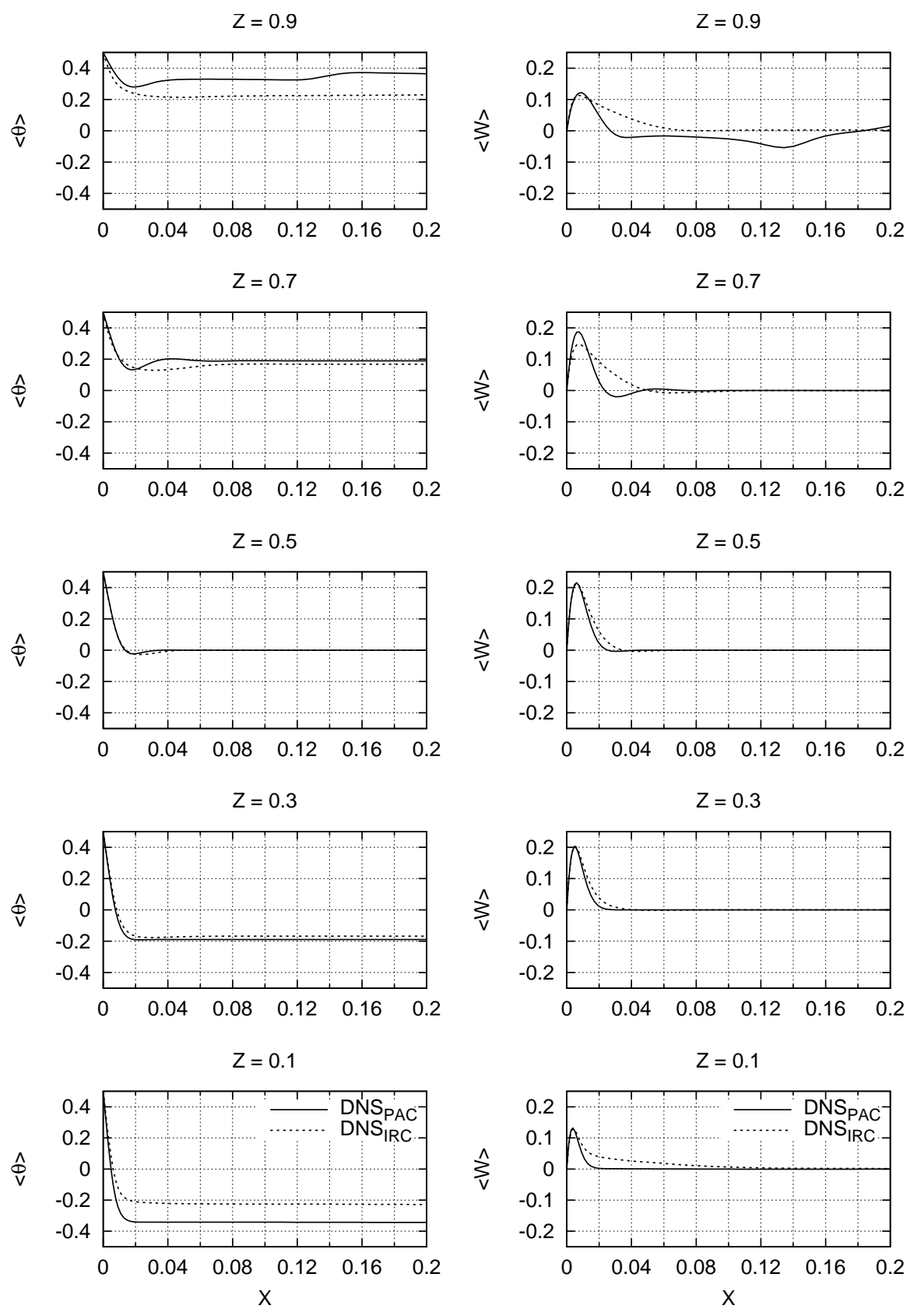

Fig. 5. Horizontal profiles of temperature $(\langle\theta\rangle)$ and vertical velocity $(<W>)$ at various $z$ positions and $y=A_{y} / 2$. Comparison between PAC and IRC.

The existence of the horizontal separating fluid layers in the PAC modifies the $U$-velocity vertical profile (Figure 4$)$ at the cavity mid-width $(x=$ $A_{x} / 2$ ) which is located downstream of the second re-attachment point. It also modifies the horizontal profiles at $z=0.9$ of the temperature and the vertical velocity $(\langle W\rangle)$ with the presence of the first separated 

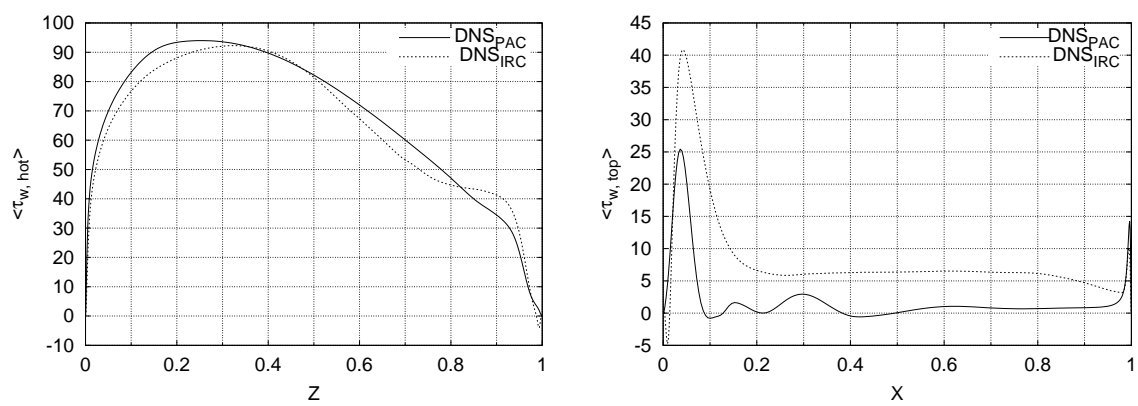

Fig. 6. iTime-averaged wall shear stress on the hot vertical wall (left) and on the top horizontal wall (right) in the mid-depth plane $\left(y=A_{y} / 2\right)$. Comparison between PAC and IRC.

region (Figure 5).

\subsection{Turbulent transition and instantaneous fields}

Considering Figure 5, we note for the IRC at $z=0.7$ a thickening of the thermal and viscous boundary layers associated with a weaker counterflow by comparison with the PAC. Moreover the IRC $W$-velocity profile decreases nearly monotonically from a velocity maximum lower than the PAC peak. This indicates that the IRC cavity gives rise to an early turbulent transition of the vertical boundary layers. This is in good agreement with the vertical distribution of the mean wall shear stress (Figure 6), which exhibits the classical laminar shape almost over the whole height of the PAC cavity, whereas the wall shear stress is reduced earlier in the downstream part of the IRC vertical boundary layer by the turbulent transition (Trias et al., 2010a).

The instantaneous fields of temperature and horizontal velocity component $(\langle U\rangle)$ at the cavity mid-depth are displayed in Figure 7 . This figure confirms the very weak flow and the uniform thermal stratification of the PAC cavity core observed by Trias et al. (2007) in their threedimensional simulations. Moreover the laminar-turbulent transition can be approximatively located at a height $z \sim 0.8$, which also agrees well with their results. Unlike PAC, the IRC top wall is colder than the hot fluid coming from the upward vertical boundary layer and this results in a locally thermally unstable boundary layer, where small scale thermal plumes develop. These fluctuations are fed into the vertical downward boundary layer and trigger a much earlier transition to turbulence for IRC (with respect to PAC). It should furthermore be noted that the presence of vortices along the vertical walls indicates a transition loca- 

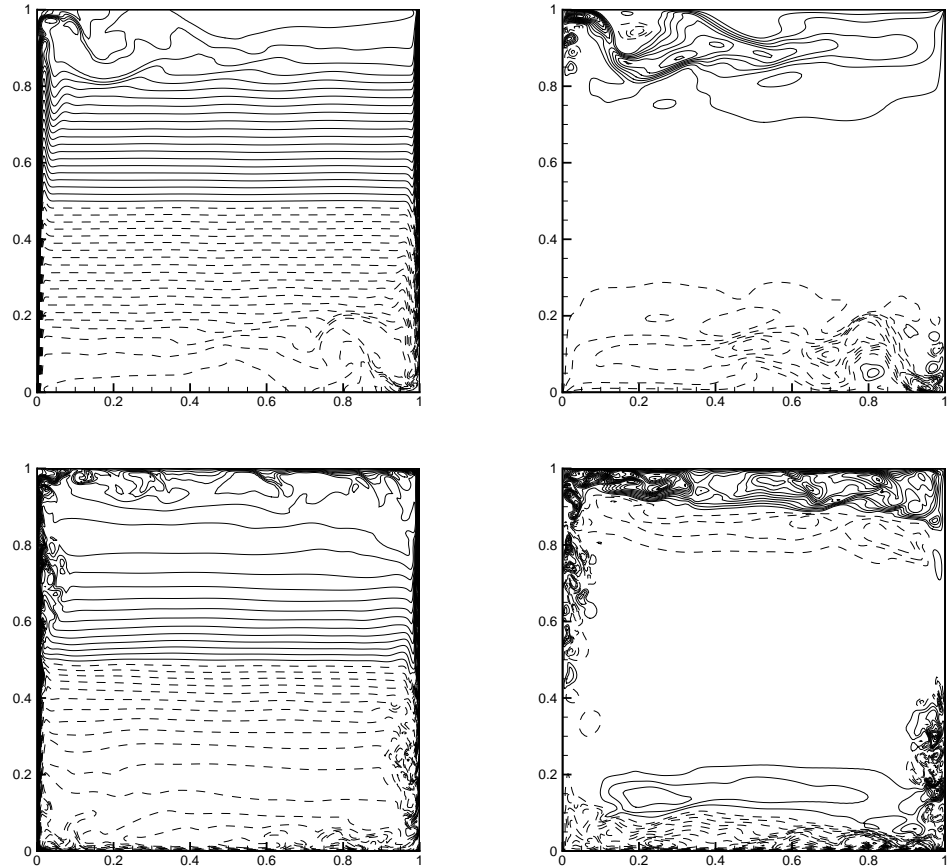

Fig. 7. Instantaneous fields of temperature $\theta$ (left) and horizontal velocity $u$ (right) in the vertical mid-depth plane $\left(y=A_{y} / 2\right)$. PAC (top) and IRC (bottom).

tion at around the mid-height of cavity $(z \sim 0.5)$.

Figure 8 depicts the coherent structures in the cavity, and especially those belonging to the boundary layers. It confirms the earlier turbulent transition occurring in IRC, as well as the fully turbulent regime of the downward part of the vertical boundary layers. It also shows that PAC exhibits a strong dependence on the spanwise direction, while still displaying the reflexion symmetry. On the contrary, the IRC flow is much more homogeneous throughout the spanwise direction.

\subsection{Power spectra}

For both configurations, low frequency oscillations are observed in the cavity core as evidenced by the time evolution of temperature at the center and the corresponding power spectra shown in Figure 9. The power spectrum is normalized such that the maximum of density is equal to one. The temperature fluctuations are weak and remarkably regular in PAC while of larger amplitude and more chaotic in IRC. However a rather similar fundamental frequency is found in both cases (see Table 4) at 

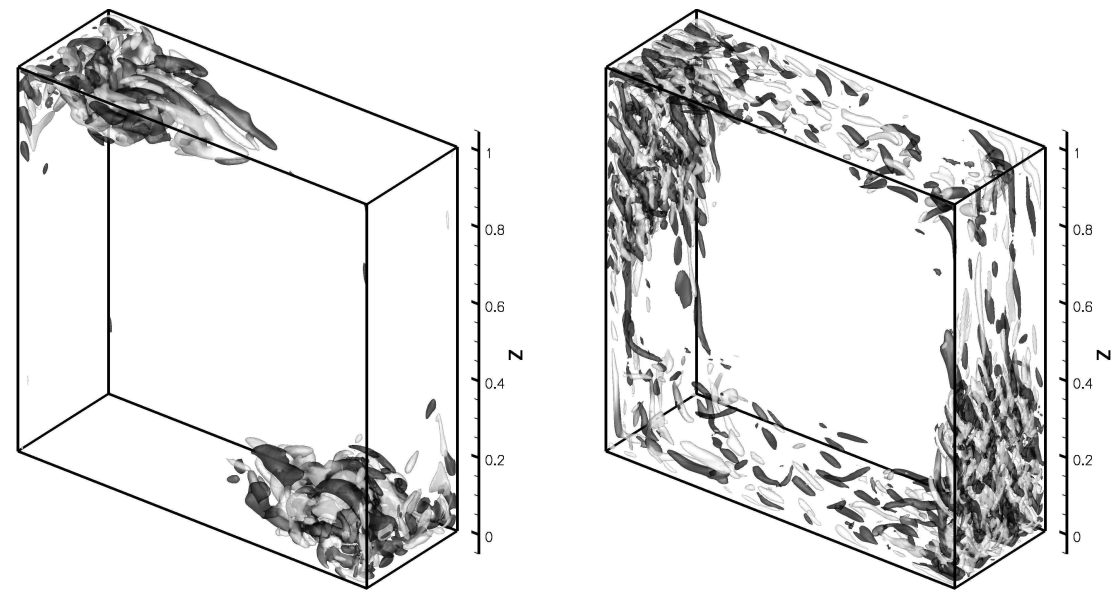

Fig. 8. Iso-surface of $\lambda_{2}$, the second eigenvalues of $\Omega_{i k} \Omega_{k j}+S_{i k} S_{k j}$ (Jeong and Hussain, 1995), colored by the vorticity component $\omega_{x}$. Left: PAC ; right: IRC.
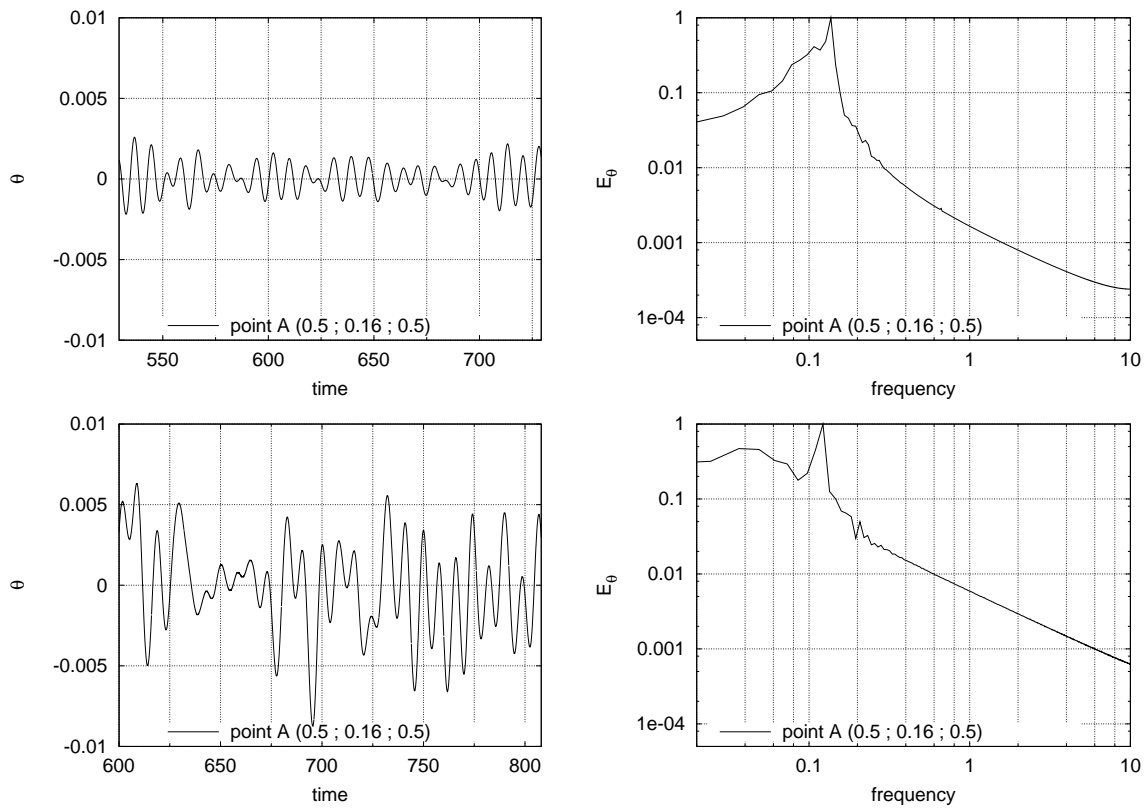

Fig. 9. Time series and normalized density power spectra of temperature at the cavity center (point A, see Table 4 for coordinates) in PAC (top) and IRC (bottom) cases.

around $f_{A} \sim 0.13$, which is in good agreement with the dimensionless Brunt-Vaïsälä frequency $N$ relating to the internal gravity waves.

The power spectra of the temperature are shown in Figure 10 for two monitoring points at the same height in the downstream part of the vertical upward boundary layer. Point (B) is located in the outer edge of the boundary layer, whereas point $(\mathrm{C})$ is located in the inner part. For each cavity, the fundamental frequencies of both locations are of the same 


\begin{tabular}{llcc} 
point $(x, y, z)$ & $D N S_{P A C}$ & $D N S_{I R C}$ \\
\hline Brunt-Vaïsälä frequency $(N=\sqrt{S P r} /(2 \pi))$ & 0.134 & 0.134 \\
\hline $\mathrm{A} \quad\left(0.5, A_{y} / 2,0.5\right)$ & 0.137 & 0.122 \\
$\mathrm{~B} \quad\left(0.14, A_{y} / 2,0.9755\right)$ & 0.078 & 0.073 \\
$\mathrm{C} \quad\left(0.017, A_{y} / 2,0.8535\right)$ & 0.664 & 0.512 \\
\hline
\end{tabular}

Table 4

Fundamental frequencies at the monitoring locations for PAC and IRC cases.
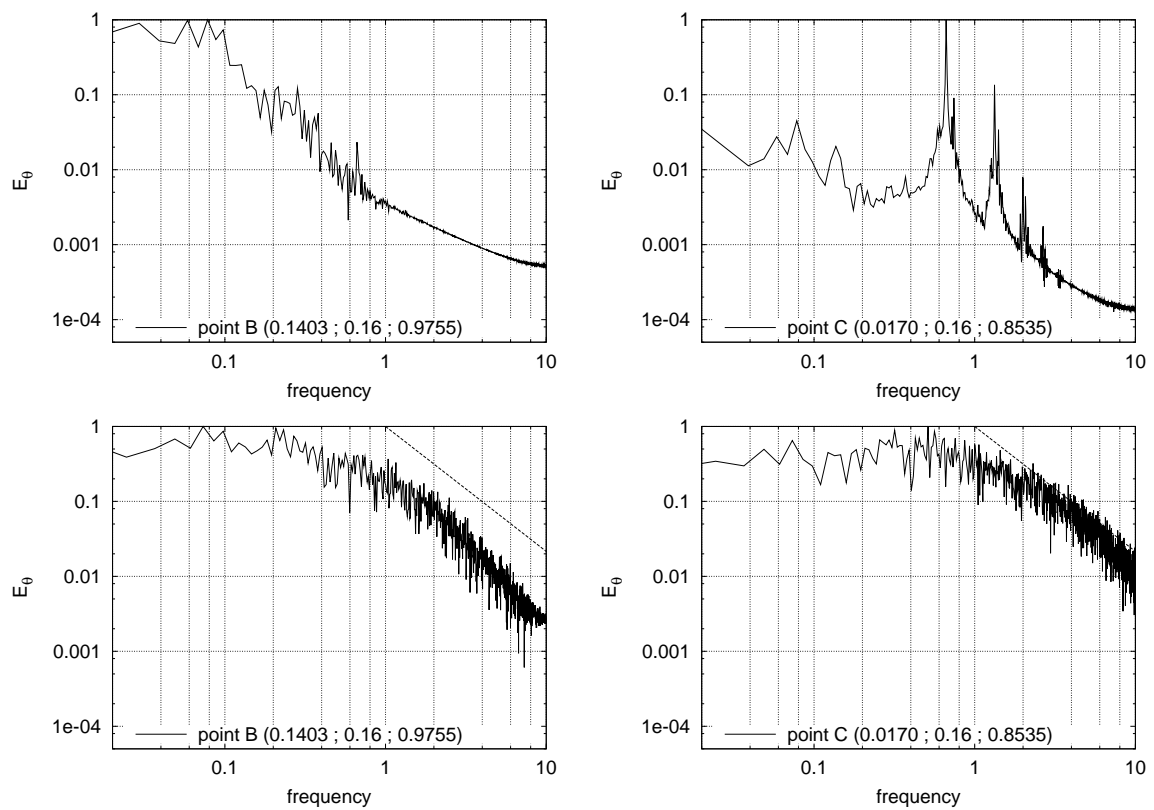

Fig. 10. Normalized density power spectra of temperature at two different locations (points B and C, see Table 4 for coordinates) in PAC (top) and IRC (bottom) cases. The Kolmogorov slope is reported for comparison (dashed line).

order. In the outer edge of the boundary layer (point B), the fundamental frequency is very weak (Table 4). It is representative of the large eddies ejected from the downstream part of the vertical boundary layers. As expected for the PAC (Xin and Le Quéré, 1995; Tian and Karayiannis, 2000), these large structures resulting from the separating fluid layer are characterized by a frequency smaller than $N$. Concerning the IRC, the value of $f_{B}$ corresponds to the base frequency of the temperature fluctuations measured by Tian and Karayiannis (2000) in the outer edge of the boundary layer.

In the inner part of the boundary layer (point $\mathrm{C}$ ), a high frequency peak is observed, corresponding to travelling waves. For the PAC, this peak at 
high frequency and its harmonics are visible in the power spectrum. This discontinuity in frequency of the spectrum is characteristic of a chaotic regime. Concerning the IRC, the power spectra are continuous for points (B) and (C) and exhibit a $-5 / 3$ slope for the highest frequencies, which indicates that the regime if fully turbulent in the vertical boundary layer.

\subsection{Second-order statistics}

\subsubsection{Qualitative analysis}

It has been shown in section 3.3 that in both configurations, the turbulence level is too low to impact the global Nusselt number $\left.\left(<\overline{N u}_{2 D}\right\rangle\right)$ : it keeps a scaling law which is characteristic of the laminar regime. In fact the major part of the cavity flow remains laminar mainly in the core. This can be observed in Figure 11 displaying the spatial distributions of the turbulent kinetic energy $(k)$, the rms fluctuations of temperature $\left(\theta_{r m s}\right)$ and the horizontal turbulent heat flux $\left.\left(<u^{\prime} \theta^{\prime}\right\rangle\right)$ in the mid-depth plane. In both cases a centro-symmetry with respect to the cavity center is exhibited. However the PAC spatial distribution of the fluctuations differs substantially from IRC. In PAC, the turbulent fluctuations are concentrated in the horizontal separating fluid layers. This does not agree with the recent results of Trias et al. (2010b), but it is explained by the fact that they considered a cavity of aspect ratio 4 which does not allow for the existence for a clearly defined detached region. The contour plot of $\left.<u^{\prime} \theta^{\prime}\right\rangle$ (Figure 3) shows the mixing effect of the successive detachments and re-attachments of the horizontal boundary layers, which make the top and bottom areas more uniform in temperature than the stratified core. In IRC the turbulent fluctuations are located along the four walls and predominantly along the vertical isothermal walls. The comparison with the experimental data Tian and Karayiannis (2000) shows that the IRC configuration allows one to reproduce the spatial distributions of the turbulent intensities. The dissimilarity of the $k$ and $\theta_{r m s}$ is indeed also observed experimentally as well as the abrupt growth located around the first quarter downstream of the vertical boundary layers. This height corresponds to the separating point between the horizontal flow reversals and the vertical boundary layers (Figure 3). 


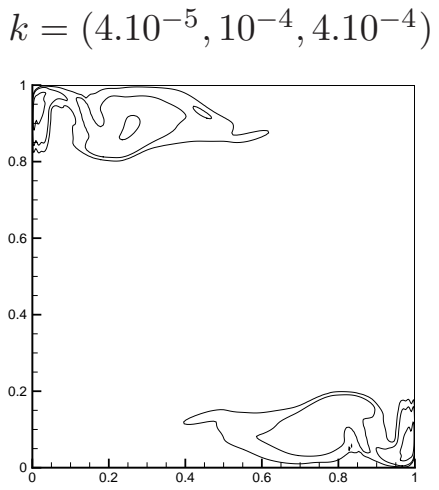

$k=\left(4.10^{-4}, 2.10^{-3}, 4.10^{-3}\right)$

$\theta_{r m s}=(0.005,0.01)$
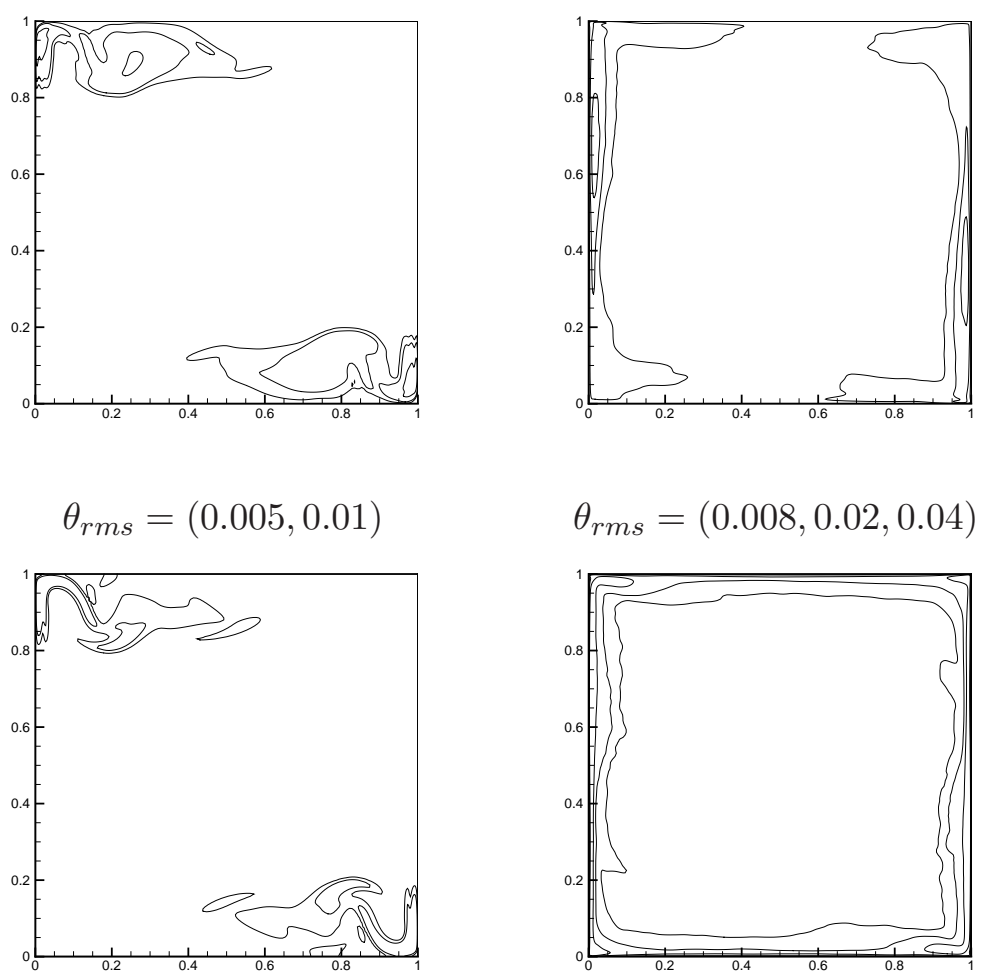

$\theta_{\text {rms }}=(0.008,0.02,0.04)$

$<u^{\prime} \theta^{\prime}>=\left( \pm 10^{-5}, \pm 10^{-4}\right)$
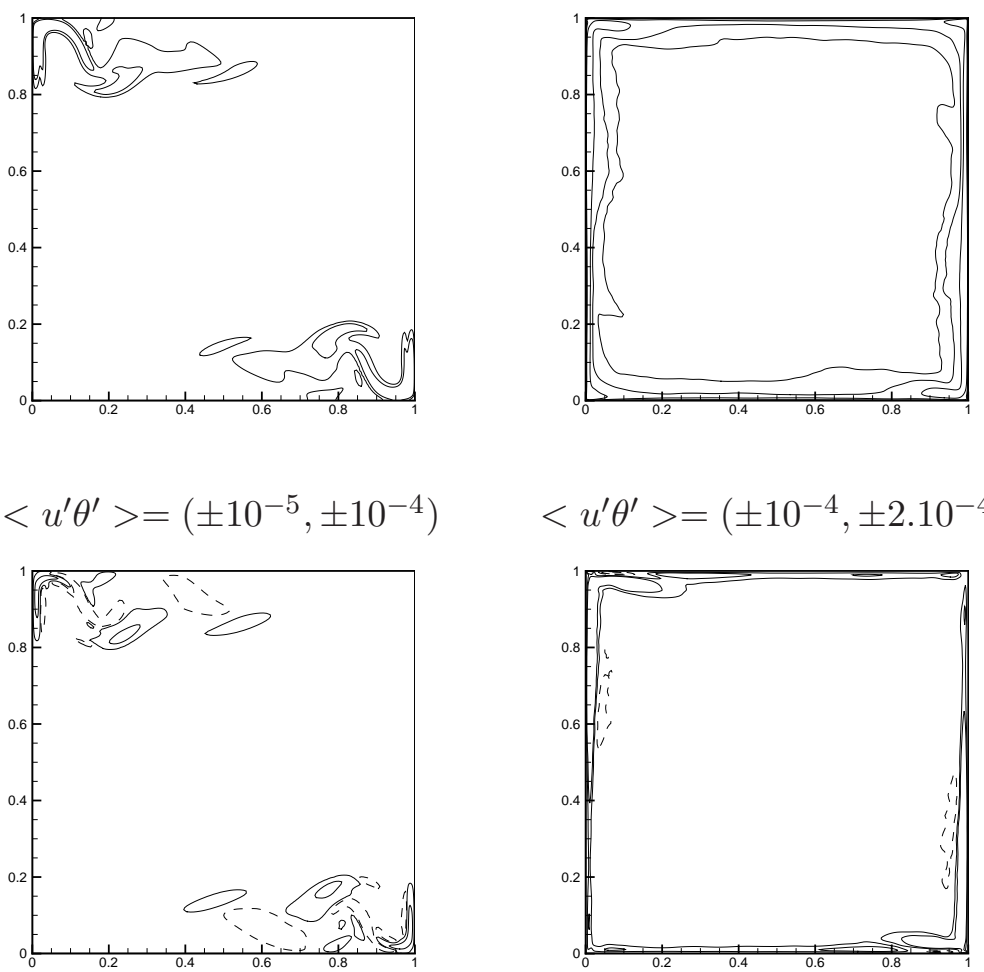

$<u^{\prime} \theta^{\prime}>=\left( \pm 10^{-4}, \pm 2.10^{-4}\right)$

Fig. 11. Isocontours of the turbulent kinetic energy $k=<u_{i}^{\prime} u_{i}^{\prime}>$ (top), the rms fluctuations of temperature $\theta_{r m s}$ (middle) and the horizontal turbulent heat flux $\left\langle u^{\prime} \theta^{\prime}\right\rangle$ (bottom) in the vertical mid-depth plane $y=A_{y} / 2$. PAC (left) and IRC (right).

\subsubsection{Quantitative analysis}

A summary of the maximum values of the Reynolds stresses is given in Table 5, which confirms the different turbulent levels induced by the BCs of PAC or IRC. Only $U_{r m s}$ keeps a similar order of magnitude in both 


\begin{tabular}{cccc|cccc}
\multicolumn{7}{c|}{$D N S_{P A C}$} & \multicolumn{5}{c}{$D N S_{I R C}$} \\
$\theta_{r m s}$ & $U_{r m s}$ & $V_{r m s}$ & $W_{r m s}$ & $\theta_{r m s}$ & $U_{r m s}$ & $V_{r m s}$ & $W_{r m s}$ \\
\hline \multicolumn{7}{c}{ Maximum of the rms fluctuations on the entire volume } \\
0.0375 & 0.0226 & 0.0191 & 0.0257 & 0.0843 & 0.0306 & 0.0483 & 0.0675 \\
$x=0.0128$ & 0.0218 & 0.0218 & 0.0049 & $x=0.0076$ & 0.0272 & 0.0245 & 0.0128 \\
$y=0.1857$ & 0.1650 & 0.0116 & 0.1650 & $y=0.1805$ & 0.1546 & 0.2853 & 0.2305 \\
$z=0.9619$ & 0.9704 & 0.9524 & 0.9263 & $z=0.6091$ & 0.7129 & 0.9960 & 0.5392 \\
\hline & Maximum of the rms fluctuations on the mid-depth plane $\left(y=A_{y} / 2\right)$ & 0.0422 & 0.0583 \\
0.0374 & 0.0226 & 0.0075 & 0.0257 & 0.0789 & 0.0288 & 0.028 \\
$x=0.0128$ & 0.0218 & 0.2350 & 0.0049 & $x=0.0076$ & 0.0245 & 0.0245 & 0.0128 \\
$z=0.9649$ & 0.9704 & 0.9045 & 0.9263 & $z=0.6470$ & 0.7545 & 0.9960 & 0.6319 \\
\hline Table 5
\end{tabular}

Table 5

Maximum of rms fluctuations of temperature and velocity components for PAC and IRC cases.

cavities. As PAC exhibits fluctuations related to the horizontal separating fluid layers, all the velocity rms fluctuations maxima have the same order of magnitude. They are located close to the top-left or bottom-right corners, and in the mid-depth vertical plane except for $V_{r m s}$, which is very weak in this plane. On the contrary, the IRC rms fluctuations are characteristic of a wall turbulence, with a vertical component $\left(W_{r m s}\right)$ larger than the two other components, which have the same order of magnitude. The $W_{r m s}$ and $\theta_{r m s}$ maxima are located around the turbulent transition point of the vertical boundary layers.

\subsubsection{Horizontal profiles in the hot boundary layer}

In order to describe in details the PAC and IRC spectral solutions, the horizontal profiles of temperature and velocities rms fluctuations at different heights are plotted in Figures 12 and 13. There are no visible rms fluctuations upstream from the PAC vertical boundary layers which remain laminar. But at $z=0.9$, two peaks on the rms profiles are observed: one in the downstream corner of the vertical boundary layer, and another resulting from the first recirculating region $(x>0.125)$. On the contrary, the fluctuations exist over the whole height of the IRC vertical boundary layers. Their peak intensities increase until the turbulent transition height $(z \leq 0.5)$. At $z=0.7$, the profiles do not decrease strictly monotonously: two successive inflection points are noticeable for the $\theta_{r m s}$ 

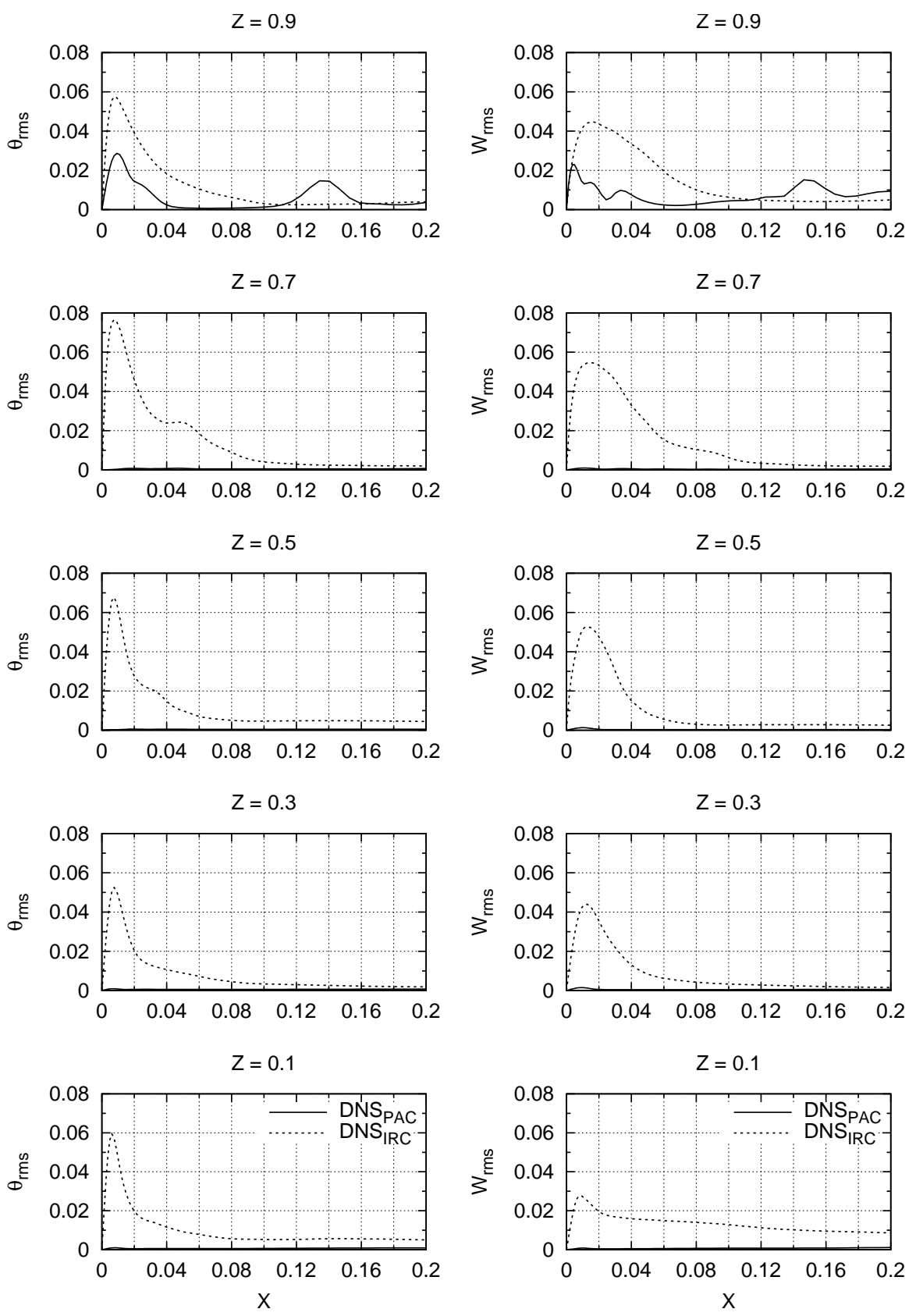

Fig. 12. Horizontal profiles of the rms fluctuations of temperature $\left(\theta_{r m s}\right)$ and vertical velocity $\left(W_{r m s}\right)$ at various $z$ positions and $y=A_{y} / 2$. Comparison between PAC and IRC.

or $W_{\text {rms }}$ profiles. This feature has been also observed experimentally by Tian and Karayiannis (2000). Figure 13 shows that at this height the peak of $U_{r m s}$ and $V_{r m s}$ is smoother or even becomes a plateau. 

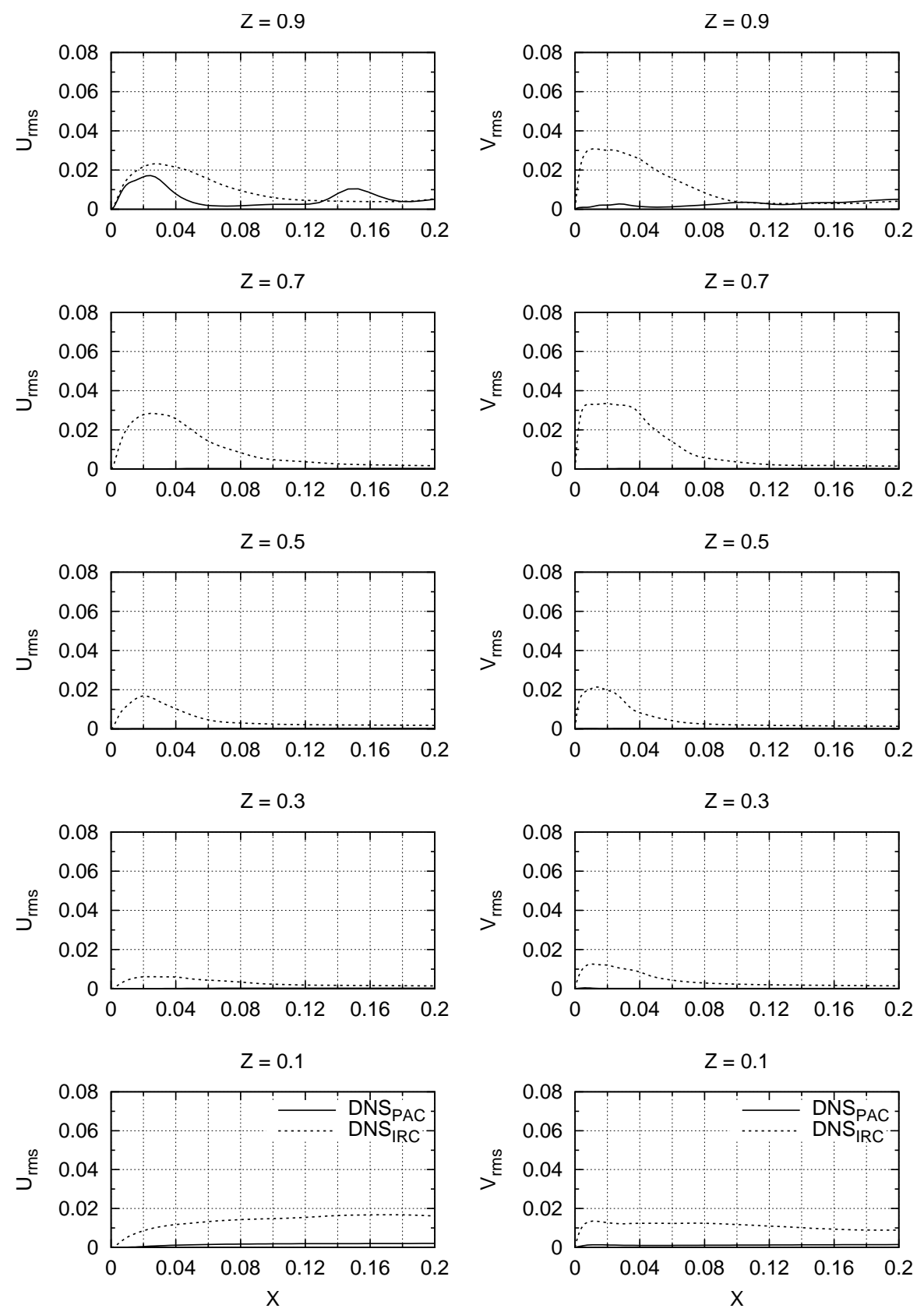

Fig. 13. Horizontal profiles of the rms fluctuations of the horizontal velocities $U_{r m s}$ and $V_{r m s}$ at various $z$ positions and $y=A_{y} / 2$. Comparison between PAC and IRC.

\section{Conclusion}

Three-dimensional direct numerical simulations of a buoyancy-driven flow in a differentially heated air-filled $(\operatorname{Pr}=0.71)$ cavity of aspect ratios 
$\left(A_{x}=1, A_{y}=0.32\right)$ at a Rayleigh number equal to $1.5 \times 10^{9}$ have been presented. Two simulations have been performed using either adiabatic conditions (PAC) or experimentally measured temperature distribution (Salat, 2004) on the top and bottom walls (IRC), while the front and rear walls were assumed to be adiabatic. The present simulations of PAC and IRC have been performed with a Chebyshev spectral collocation approximation. The combination of very large spatial resolution and long time integration guarantees the quality of the present results. The aim of this work is to provide reference results in order to separate the potential reasons (numerical errors, unsuitable physical or turbulence models) responsible for the long established discrepancy in thermal stratification observed between experimental and numerical estimates using either DNS, LES or RANS simulations.

Noteworthy is the modification of the flow structure by the thermal BCs in the horizontal walls. PAC flow structures are characterized by thin vertical boundary layers along the isothermal walls and two successive separated recirculating flow regions in the upstream part of the horizontal boundary layers. Only a chaotic behavior of the vertical boundary layers has been observed. In the IRC, the flow exhibits two strong recirculating regions along the whole top and bottom walls of the cavity and turbulent vertical boundary layers in their downstream parts. This IRC flow structure as well as the relative $r m s$ fluctuations distribution are typical of the flow dynamics observed experimentally.

However both 3D simulations keep resulting in a thermal stratification value equal to one around the cavity mid-height, leading to the conclusion that neither the tridimensional effect nor the experimental distribution on the top/bottom walls improve the quality of numerical prediction concerning the thermal stratification. Moreover, the IRC simulation reveals that the time-averaged results in the vertical mid-depth plane are in good agreement with the previous $2 \mathrm{D}$ results. This was also observed by Soria et al. (2004) and Trias et al. (2007) concerning the general features of the flow when performing 2D and 3D DNS with periodicity in the spanwise direction in an adiabatic cavity (PAC) of aspect ratio 4 for a Rayleigh number up to $10^{10}$. This confirms, in agreement with Fusegi and Hyun (1994), that realistic thermal BCs on the top and bottom walls are not key factors for explaining the weak vertical stratification observed in the experimental studies.

The conclusion that can be drawn from these results is that both configurations miss a physical phenomenon that prevents from recovering the experimental data. The influence of the thermal BCs applied on the front 
and rear walls will be the subject of the second part of the paper (Sergent et al., 2010).

\section{Acknowledgments}

Support by the energy program (COCORAPHA and COCORACOPHA) from CNRS, the french National Center for Scientific Research, is gratefully acknowledged. Computations have been performed at IDRIS (Institute for Development and Resources in Intensive Scientific computing) under projects 70327 and 0326.

\section{References}

Achdou, Y., Guermond, J., 2000. Convergence analysis of a finite element projection/lagrange-galerkin method for the incompressible navierstokes equations. SIAM J. Numer. Anal. 37, 799-826.

Baraghi, D. G., Davidson, L., 2007. Natural convection boundary layer in a 5:1 cavity. Phys. Fluids 19, 1-15.

de Gassowski, G., Daube, O., Xin, S., Fraigneau, Y., 2006. Bifurcations and multiple solutions in an air-filled differentially heated cubic cavity. IHTC-13. 13th International Heat Transfer Conference. Sydney, Australia, pp. TRB-05_1-TRB-05_12.

de Gassowski, G., Xin, S., Daube, O., 2003. Bifurcations et solutions multiples en cavité 3d différentiellement chauffée. C. R. Mécanique (331), $705-711$.

de Vahl Davis, G., Jones, I. P., 1983. Natural convection in a square cavity: a comparison exercise. Int. J. Numer. Meth. Fluids 3, 227-248.

Dol, H. S., Hanjalić, K., 2001. Computational study of turbulent natural convection in a side-heated near-cubic enclosure at a high rayleigh number. Int. J. Heat Mass Transfer 44, 2323-2344.

Fusegi, T., Hyun, J. M., 1994. Laminar and transitional natural convection in an enclosure with complex and realistic conditions. International Journal of Heat and Fluid Flow 15 (4), 258 - 268.

Fusegi, T., Hyun, J. M., Kuwahara, K., 1993. Three-dimensional natural convection in a cubical enclosure with walls of finite conductance. International Journal of Heat and Mass Transfer 36 (7), 1993 - 1997.

Guermond, J. L., Quartapelle, L., 1998. On stability and convergence of projection methods based on pressure equation. Int. J. Numer. Meth. Fluids 26, 1039-1053. 
Henkes, R. A. W. M., Hoogendoorn, C. J., 1992. Turbulent natural convection in enclosures; a computational and experimental benchmark study. Proc. ERCOFTAC/EUROTHERM Sem. 22, Delft. EETI, Paris.

Henkes, R. A. W. M., Hoogendoorn, C. J., 1995. Comparison exercise for computations of turbulent natural convection in enclosures. Num. Heat Transfer Part. B 28, 59-78.

Janssen, R. J. A., Henkes, R. A. W. M., 1996. Instability in threedimensional differentially-heated cavities with adiabatic horizontal walls. Phys. Fluids 8 (1), 62-74.

Jeong, J., Hussain, F., 1995. On the identification of a vortex. J. Fluid Mech. 285, 69-94.

Labrosse, G., Tric, E., Khallouf, H., Betrouni, M., 1997. A direct (pseudospectral) solver of the $2 \mathrm{~d} / 3 \mathrm{~d}$ stokes problem: transition to unsteadiness of natural convection flow in a differentially heated cubical cavity. Num. Heat Transfer Part. B 31, 261-276.

Le Quéré, P., 1991. Accurate solutions to the square thermally driven cavity at high rayleigh number. Comput. Fluids 20, 29-41.

Le Quéré, P., Alziary de Roquefort, T., 1985. Computation of natural convection in two-dimensional cavities with Chebyshef polynomials. J. Comp. Phys. 57 (2), 210-228.

Le Quéré, P., Behnia, M., 1998. From onset of unsteadiness to chaos in a differentially heated square cavity. J. Fluid Mech. 359, 81-107.

Mallinson, G. D., de Vahl Davis, G., 1977. Three-dimensional natural convection in a box: a numerical study. J. Fluid Mech. 83 (1), 1-31.

Markatos, N. C., Pericleous, K. A., 1984. Laminar and turbulent natural convection in an enclosed cavity. Int. J. Heat Mass Transfer 27, 755772 .

Mergui, S., Penot, F., 1997. Analyse des vitesse et température de l'air en convection naturelle dans une cavité carrée différentiellement chauffée à $R a=1.69 \times 10^{9}$. Int. J. Heat Mass Transfer 40, 3427-3441.

Nobile, E., 2002. Simulation of time-dependent flow in cavities with the additive-correction multigrid method, part II: applications. Num. Heat Transfer Part. B 30, 351-370.

Paolucci, S., 1990. Direct simulation of two dimensional turbulent natural transition in an enclosed cavity. J. Fluid Mech. 215, 229-262.

Peng, S., Davidson, L., 2001. Large eddy simulation for turbulent buoyant flow in a confined cavity. Int. J. Heat Fluid Flow 22, 323-331.

Salat, J., 2004. Contribution à l'étude de la convection naturelle tridimensionnelle en cavité différentiellement chauffée. Ph.D. thesis, Université de Poitiers.

Salat, J., Xin, S., Joubert, P., Sergent, A., Penot, F., Le Quéré, P., 2004. Experimental and numerical investigation of turbulent natural convec- 
tion in a large air-filled cavity. Int. J. Heat Fluid Flow 25 (5), 824-832.

Sergent, A., Joubert, P., Le Quéré, P., 2003. Development of a local subgrid diffusivity model for large eddy simulation of buoyancy driven flows: application to a square differentially heated cavity. Num. Heat Transfer Part. A 44 (8), 789-810.

Sergent, A., Joubert, P., Xin, S., Le Quéré, P., Salat, J., Penot, F., 2010. Turbulent natural convection in differentially heated air-filled cavities at $R a=1.510^{9}$. Part II: resolving the stratification discrepancy. Int. J. Heat Fluid Flow, submitted.

Soria, M., Trias, F. X., Pérez-Ségarra, C. D., Oliva, A., 2004. Direct numerical simulation of a three-dimensional natural convection flow in a differentially heated cavity of aspect ratio 4. Num. Heat Transfer Part. A 45, 649-673.

Tian, Y., Karayiannis, T., 2000. Low turbulence natural convection in an air filled square cavity, part I \& II. Int. J. Heat Mass Transfer 43, 849-884.

Trias, X., Gorobets, A., Soria, M., Oliva, A., 2010a. Direct numerical simulation of a differentially heated cavity of aspect ratio 4 with Rayleigh numbers up to $10^{11}$ - part I: Numerical methods and time-averaged flow. Int. J. Heat Mass Transfer 53, 665-673.

Trias, X., Gorobets, A., Soria, M., Oliva, A., 2010b. Direct numerical simulation of a differentially heated cavity of aspect ratio 4 with Rayleigh numbers up to $10^{11}$ - part II: heat transfer and flow dynamics. Int. J. Heat Mass Transfer 53, 674-683.

Trias, X., Soria, M., Oliva, A., Pérez-Ségarra, C. D., 2007. Direct numerical simulation of two and three-dimensional natural convection flows in a differentially heated cavity of aspect ratio 4. J. Fluid Mech. 586, 259-293.

Tric, E., Labrosse, G., Betrouni, M., 2000. A first incursion into the 3d structure of natural convection of air in a differentially heated cubic cavity, from accurate numerical solutions. Int. J. Heat Mass Transfer 43, 4043-4056.

Xin, S., Joubert, P., Sergent, A., Le Quéré, P., 2010. Turbulent natural convection in differentially heated air-filled cavities. Part III: a full convection-conduction-surface radiation coupling. Int. J. Heat Fluid Flow, submitted.

Xin, S., Le Quéré, P., 1995. Direct numerical simulations of twodimensional chaotic natural convection in a differentially heated cavity of aspect ratio 4. J. Fluid Mech. 304, 87-118.

Xin, S., Le Quéré, P., 2002. An extended chebyshev pseudo-spectral benchmark for the 8:1 differentially heated cavity. Int. J. Numer. Meth. Fluids 40 (8), 981-998. 
Yang, K. T., Novotny, J. L., Cheng, Y. S., 1972. Laminar free convection from a non-isothermal plate immersed in a temperature stratified medium. Int. J. Heat Mass Transfer 15, 1097-1109. 